\title{
Hybrid Cobalt(II) Fluoride Derived from Bimetallic Zeolitic Imidazolate Framework as a High-Performance Cathode for Lithium-Ion Batteries
}

Qiuxia Cheng, ${ }^{\dagger}$ Yueying Chen,$^{\dagger}$ Xiaoming Lin, ${ }^{*}, \dagger, \S$ Jincheng Liu, ${ }^{\ddagger}$ Zhongzhi Yuan ${ }^{*}, \dagger$ and Yuepeng $\mathrm{Cai}^{*}, \dagger$

${ }^{\dagger}$ Guangzhou Key Laboratory of Materials for Energy Conversion and Storage, School of Chemistry, South China Normal University, Guangzhou 510006, P. R. China

${ }^{*}$ EVE Energy Co. Ltd, Huizhou, Guangdong 516006, P.R. China

$\S$ School of Environment and Energy, South China University of Technology, Guangzhou, Guangdong 510006, P.R. China

\footnotetext{
*Author to whom correspondence should be addressed. E-mail: linxm@scnu.edu.cn (X. Lin); yuanzz@scnu.edu.cn (Z. Yuan); caiyp@scnu.edu.cn (Y. Cai).
} 


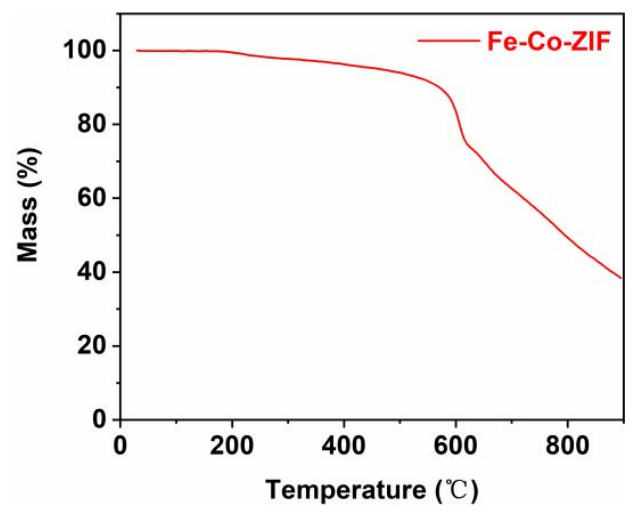

Figure S1. TG curve under $\mathrm{N}_{2}$ of Fe-Co-ZIF.

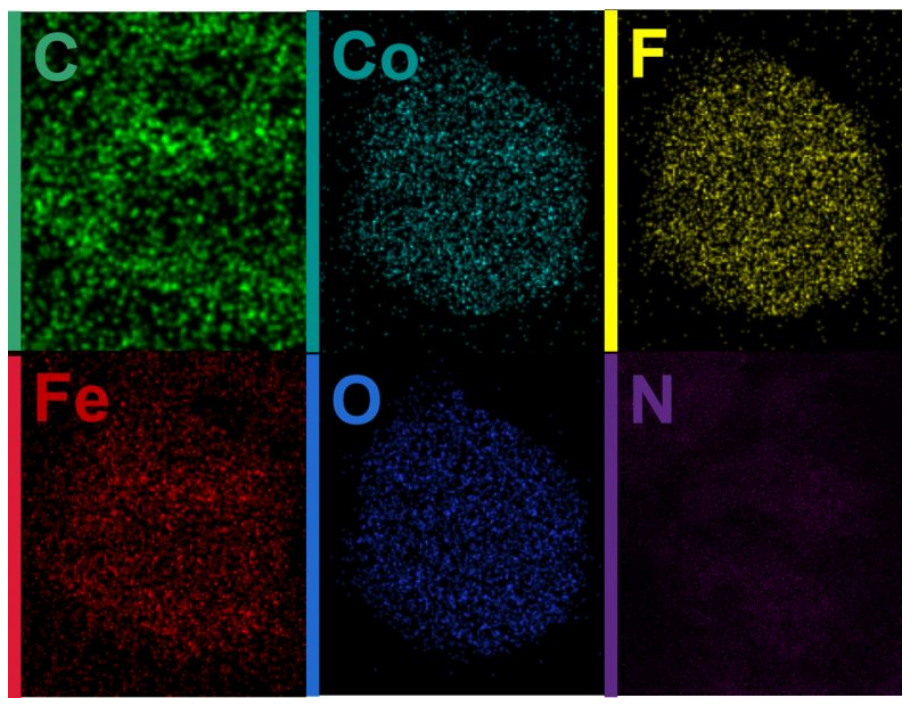

Figure S2 EDS mapping results.
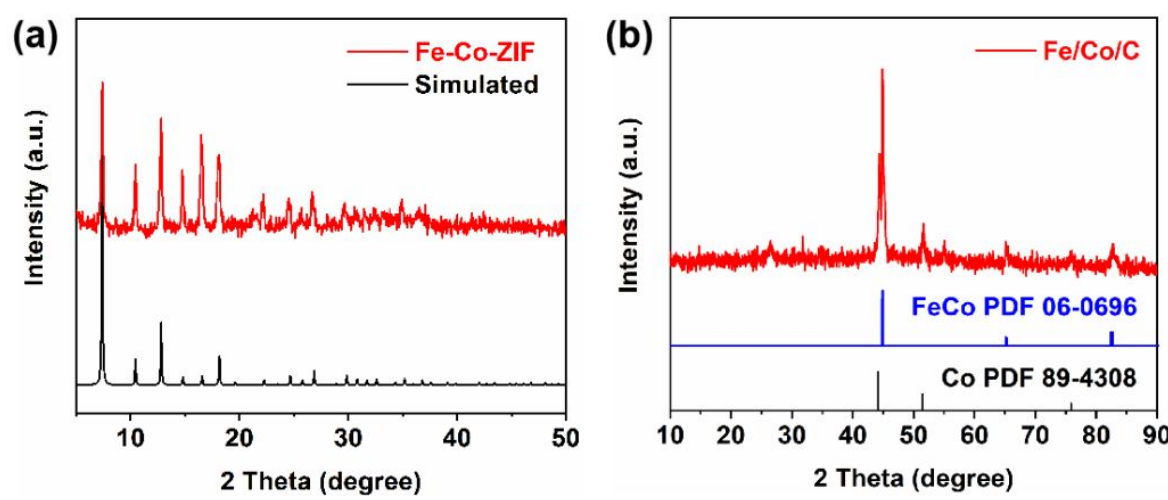

Figure S3 XRD patterns of (a) Fe-Co-ZIF, (b) $\mathrm{Fe} / \mathrm{Co} / \mathrm{C}$. 

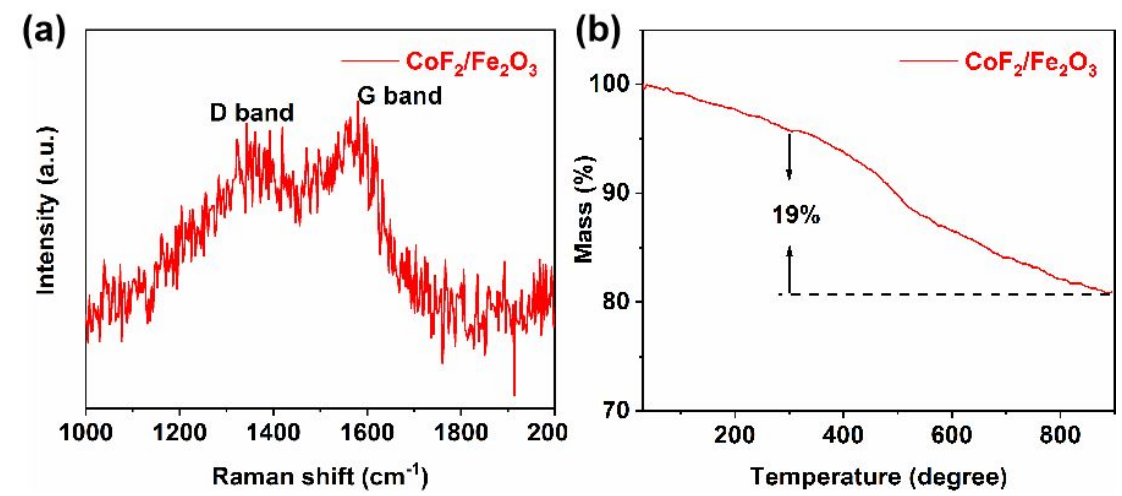

Figure S4. Characterization of $\mathrm{CoF}_{2} / \mathrm{Fe}_{2} \mathrm{O}_{3}$. (a) Raman spectrum. (b) $\mathrm{TG}$ curve in air.
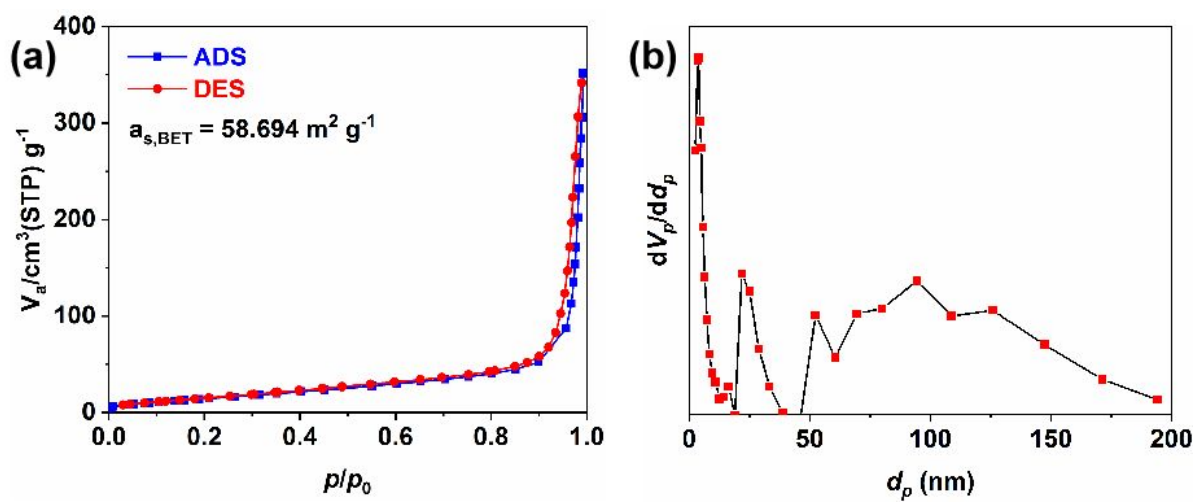

Figure S5. (a) $\mathrm{N}_{2}$ adsorption-desorption isotherm. (b) Pore size.
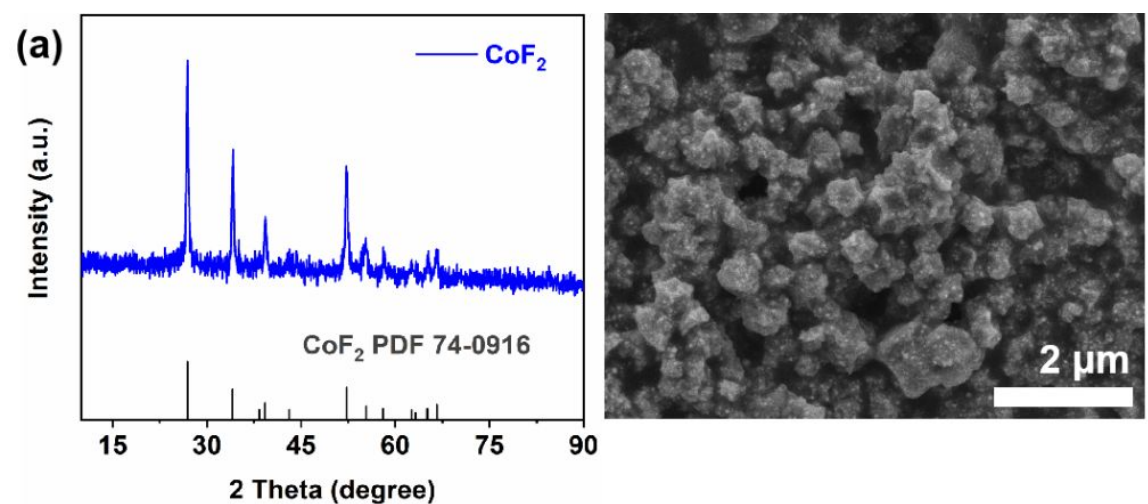

Figure S6. Characterization ZIF-derived $\mathrm{CoF}_{2}$. (a) XRD, (b) SEM image. 


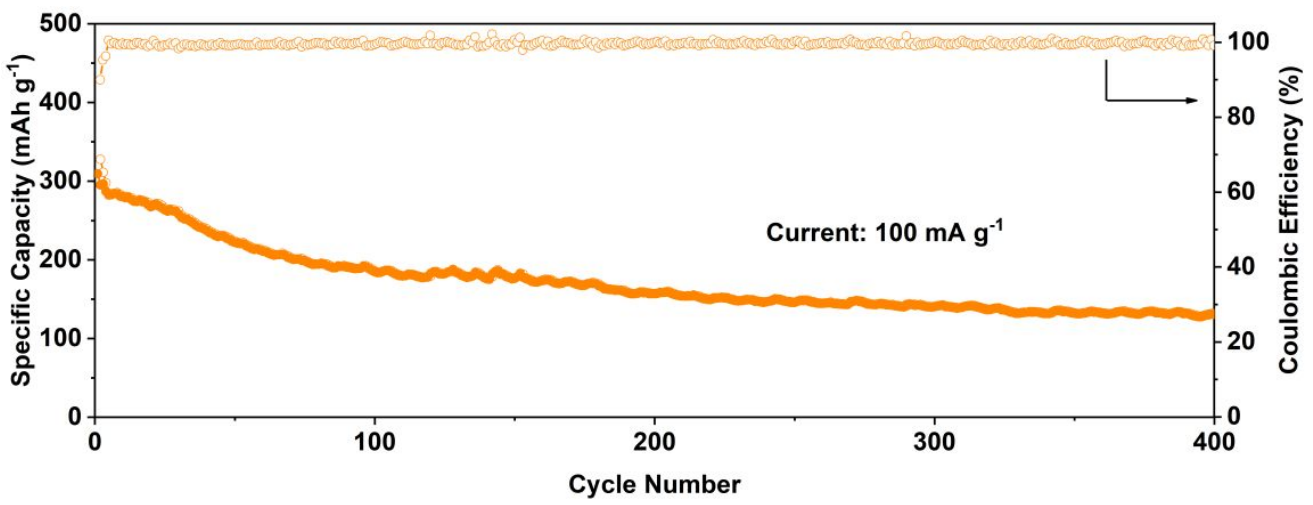

Figure S7. Cycling specific capacity and coulomb efficiency of $\mathrm{CoF}_{2} / \mathrm{Fe}_{2} \mathrm{O}_{3}$ sample.

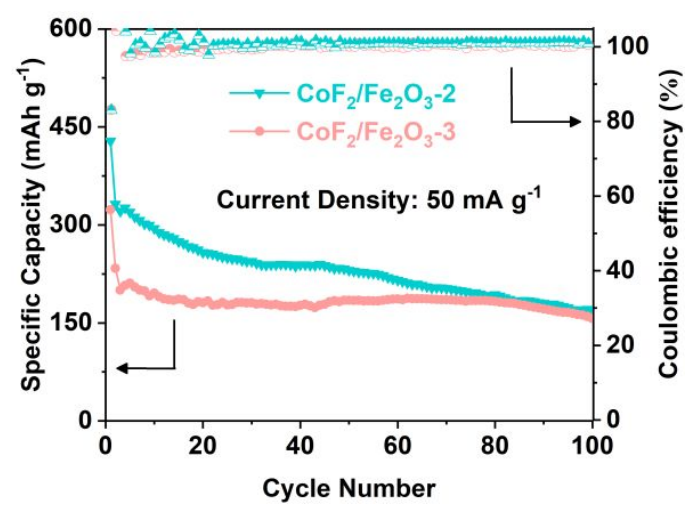

Figure S8. Electrochemical performance of $\mathrm{CoF}_{2} / \mathrm{Fe}_{2} \mathrm{O}_{3}-2$ and $\mathrm{CoF}_{2} / \mathrm{Fe}_{2} \mathrm{O}_{3}-3$. 
Table S1 Comparison of specific capacity of cobalt fluoride materials.

\begin{tabular}{cccccc}
\hline Name & $\begin{array}{c}\text { Voltage range } \\
(\mathrm{V})\end{array}$ & $\begin{array}{c}\text { Current } \\
\left(\mathrm{mA} \mathrm{g}^{-1}\right)\end{array}$ & $\begin{array}{c}\text { Cycle } \\
\text { number }\end{array}$ & $\begin{array}{c}\text { Capacity } \\
\left(\mathrm{mAh} \mathrm{g}^{-1}\right)\end{array}$ & Reference \\
\hline $\mathrm{CoF}_{2}$ & $1.0-4.5$ & 55.3 & 30 & 50 & $(\mathrm{~S} 1)$ \\
$\mathrm{CoF}_{2}(\mathrm{NC} 3100)$ & $1.0-4.3$ & 50 & 10 & 175 & $(\mathrm{~S} 2)$ \\
$\mathrm{CoF}_{2} / \mathrm{CNT}$ & $1.0-4.0$ & 100 & 200 & 334.8 & $(\mathrm{~S} 3)$ \\
$\mathrm{CoF}_{2}$ & $1.2-4.8$ & 20 & 25 & 50 & (S4) \\
$\mathrm{Fe}_{(1-\mathrm{x})} \mathrm{Co}_{\mathrm{x}} \mathrm{F}_{3} / \mathrm{MWCN}$ & $2.0-4.5$ & 47.4 & 50 & 187.9 & (S5) \\
$\mathrm{T}(\mathrm{x}=0.04)$ & $1.0-4.8$ & 20 & 30 & 127.4 & (S6) \\
$\mathrm{CoF}_{2}\left(\mathrm{~S}_{200}\right)$ & $1.2-4.5$ & 50 & 100 & 198.1 & This work \\
$\mathrm{CoF}_{2} / \mathrm{Fe}_{2} \mathrm{O}_{3}$ & & & & & \\
\hline
\end{tabular}

Table S2 $\mathrm{R}_{\mathrm{ct}}$ values of the as-prepared materials before and after cycles.

\begin{tabular}{ccc}
\hline & Fresh & After 50 cycles \\
\cline { 2 - 3 } Name & $\mathrm{R}_{\mathrm{ct}}$ & $\mathrm{R}_{\mathrm{ct}}$ \\
\hline $\mathrm{CoF}_{2}$ & $293 \Omega$ & $80 \Omega$ \\
$\mathrm{CoF}_{2} / \mathrm{Fe}_{2} \mathrm{O}_{3}$ & $196 \Omega$ & $78 \Omega$ \\
\hline
\end{tabular}




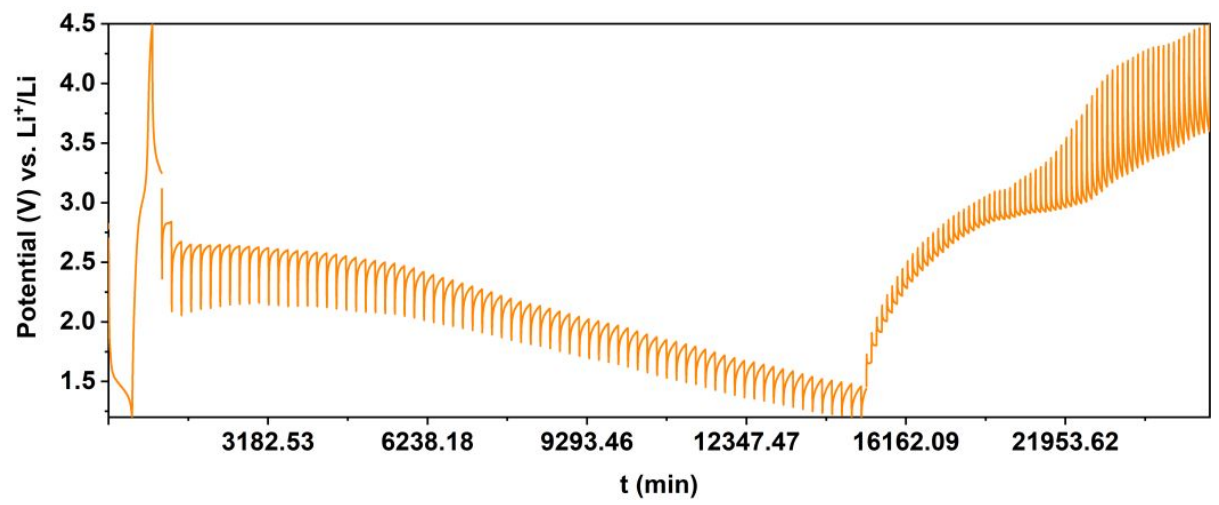

Figure S9. GITT result during the whole charge-discharge process.

Table S3 $\mathrm{D}_{\mathrm{Li}}^{+}$camparsion of various fluoride electrods.

\begin{tabular}{cccc}
\hline Method & Name & Magnitude of $\mathbf{D}_{\mathbf{L i}}{ }^{+}\left(\mathbf{m}^{\mathbf{2}} \mathbf{s}^{-1}\right)$ & Reference \\
\hline \multirow{2}{*}{ EIS } & $\mathrm{Fe}_{(1-\mathrm{x})} \mathrm{Co}_{\mathrm{x}} \mathrm{F}_{3} / \mathrm{MWCNT}$ & $10^{-12} \sim 10^{-11}$ & (S5) \\
& $\mathrm{Fe}_{1-\mathrm{x}} \mathrm{Mn}_{\mathrm{x}} \mathrm{F}_{3} \bullet 0.33 \mathrm{H}_{2} \mathrm{O} / \mathrm{C}$ & $10^{-16}$ & (S7) \\
& $\mathrm{M}-\mathrm{CF}_{\mathrm{x}}-5$ & $10^{-12} \sim 10^{-11}$ & (S8) \\
\multirow{2}{*}{ GITT } & $\mathrm{FFH}-\mathrm{P}$ & $10^{-13} \sim 10^{-12}$ & (S9) \\
& $\mathrm{CoF}_{2} / \mathrm{Fe}_{2} \mathrm{O}_{3}$ & $10^{-13} \sim 10^{-12}$ & This work \\
\hline
\end{tabular}

\section{Reference}

(S1) Tan, J.; Liu, L.; Guo, S.; Hu, H.; Yan, Z.; Zhou, Q.; Huang, Z.; Shu, H.; Yang, X.; Wang,

X. The Electrochemical Performance and Mechanism of Cobalt (II) Fluoride as Anode Material for Lithium and Sodium Ion Batteries. Electrochim. Acta 2015, 168, 225-233.

(S2) Teng, Y. T.; Pramana, S. S.; Ding, J.; Wu, T.; Yazami, R. Investigation of the Conversion Mechanism of Nanosized $\mathrm{CoF}_{2}$. Electrochim. Acta 2013, 107, 301-312. 
(S3) Wang, X. R.; Gu W. T.; Lee, J. T.; Nitta, N.; Benson, J.; Magasinski, A.; Schauer, M. W.; Yushin, G. Carbon Nanotube- $\mathrm{CoF}_{2}$ Multifunctional Cathode for Lithium Ion Batteries: Effect of Electrolyte on Cycle Stability, Small 2015, 11, 5164-5173.

(S4) Armstrong, M. J.; Panneerselvam, A.; O'Regan, C.; Morris, M. A.; Holmes, J. D. Supercritical-Fluid Synthesis of $\mathrm{FeF}_{2}$ and $\mathrm{CoF}_{2}$ Li-Ion Conversion Materials. J. Mater. Chem. A 2013, 1, 10667-10676.

(S5) Li, J.; Xu, S.; Huang, S.; Lu, L.; Lan, L.; Li, S. In Situ Synthesis of $\mathrm{Fe}_{(1-\mathrm{x})} \mathrm{Co}_{\mathrm{x}} \mathrm{F}_{3} / \mathrm{MWCNT}$ Nanocomposites with Excellent Electrochemical Performance for Lithium-Ion Batteries. J Mater Sci. 2018, 53, 2697-2708.

(S6) Guan, Q.; Cheng, J.; Li, X.; Ni, W.; Wang, B. Porous $\mathrm{CoF}_{2}$ Spheres Synthesized by a One-Pot Solvothermal Method as High Capacity Cathode Materials for Lithium-Ion Batteries. Chin. J. Chem. 2017, 35, 48-54.

(S7) Ding, J.; Zhou, X.; Wang, H.; Yang, J.; Gao, Y.; Tang, J. Mn-Doped $\mathrm{Fe}_{1-\mathrm{x}} \mathrm{Mn}_{\mathrm{x}} \mathrm{F}_{3} .0 .33 \mathrm{H}_{2} \mathrm{O} / \mathrm{C}$ Cathodes for Li-Ion Batteries: First-Principles Calculations and Experimental Study. ACS Appl. Mater. Interfaces 2019, 11, 3852-3860.

(S8) Zhou, P.; Weng, J.; Liu, X.; Li, Y.; Wang, L.; Wu, X.; Zhou, T.; Zhou, J.; Zhuo, S. Urea-Assistant Ball-Milled CF as Electrode Material for Primary Lithium Battery with Improved Energy Density and Power Density. J. Power Sources 2019, 414, 210-217.

(S9) Chen, G.; Zhou, X.; Bai, Y.; Yuan, Y.; Li, Y.; Chen, M.; Ma, L.; Tan, G.; Hu, J.; Wang, Z.; Wu, F.; Wu, C.; Lu, J. Enhanced Lithium Storage Capability of $\mathrm{FeF}_{3} \cdot 0.33 \mathrm{H}_{2} \mathrm{O}$ Single Crystal with Active Insertion Site Exposed. Nano Energy 2019, 56, 884-892. 